\title{
Nanocrystals Research for Energy Efficient and Clean Energy Technologies: DOE-BES Final Report
}

The following summarizes projects completed or in the process of being completed during the final year of funding in 2008. Efforts centered on: nanocrystal photovoltaic fabrication, ultrafast dynamics and aberration-corrected STEM characterization of II-VI core, core/shell and alloyed nanocrystals, and fundamental investigation and applications of ultrasmall white light-emitting CdSe nanocrystal.

\section{Photovoltaic Cells fabricated by electrophoretic deposition of CdSe nanocrystals}

Electrophoretic deposition (EPD) was investigated as a method for depositing nanocrystals onto counter electrodes for photovoltaic devices. ${ }^{1}$ The deposition current followed an exponential decay, consistent with the current being comprised of charge transfer from charged nanocrystals to the electrodes. Rutherford backscattering spectroscopy indicated the composition of the deposited films depended on the polarity of the electrode on which the film formed. EPD deposition was used to construct a simple device, which exhibited a photovoltaic response from the region in which nanocrystals were deposited, with an efficiency of $\sim 10^{-6} \%$. The low efficiency was likely due to the planar geometry employed for the $\mathrm{TiO}_{2}$ substrate.
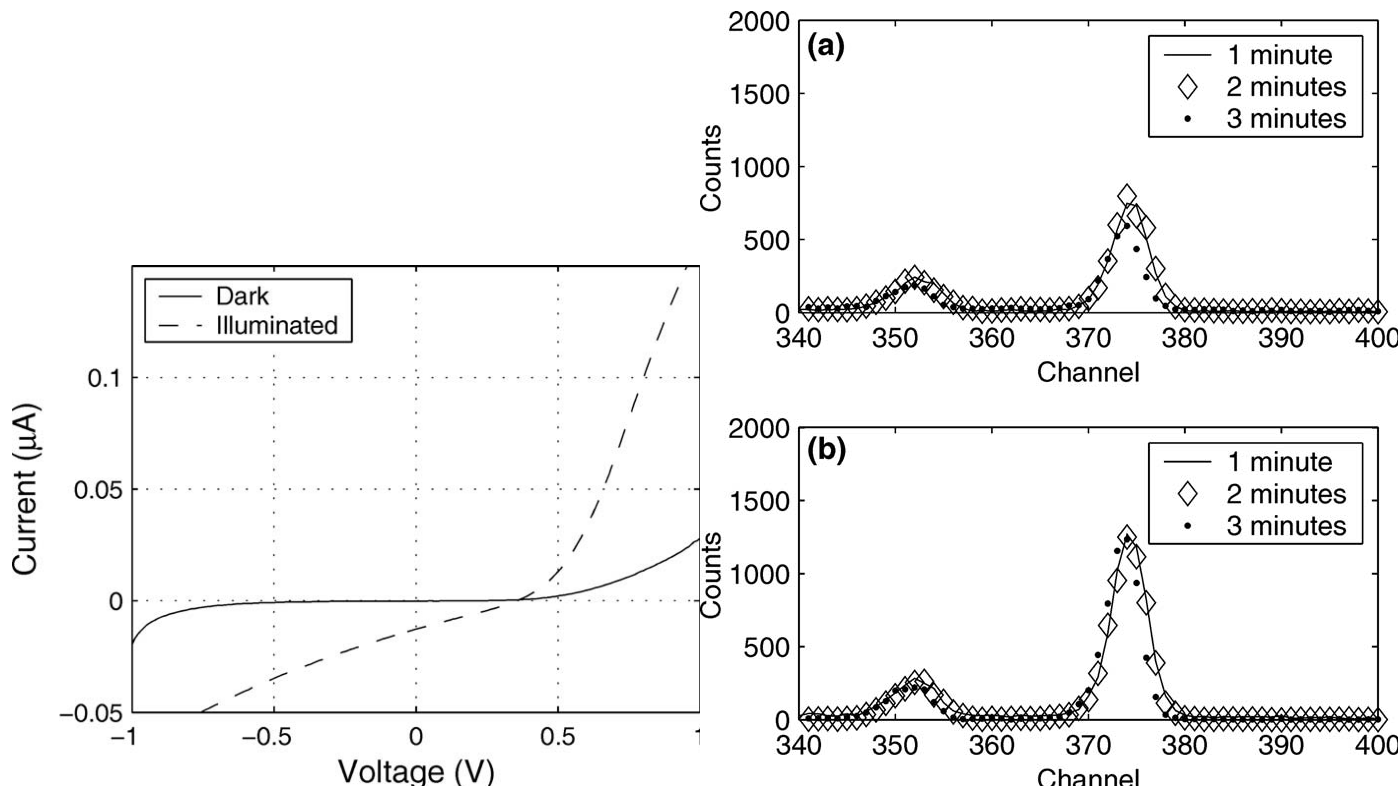

Figure 1. Fabrication of nanocrystal photovoltaic using EPD. Left: current vs. voltage curve showing photovoltaic response. Right: Rutherford backscattering spectra showing electrode deposition dependence. ${ }^{1}$ 


\section{Band Edge Dynamics in CdSe Nanocrystals Observed by Ultrafast Fluorescence Upconversion}

The change in carrier dynamics of cadmium chalcogenide nanocrystals associated with varying ligands and surface composition has been explored through femtosecond fluorescence upconversion. ${ }^{2}$ Exploring fluorescence lifetime at the band edge for CdSe nanocrystals, it was revealed that both the short- and long-lived components $\left(\tau_{1}\right.$ and $\tau_{2}$, respectively) are size dependent (Figure 2a). Specifically, it was shown that an increase in nanocrystal size is accompanied by an increase in $\tau_{1}$. The change in CdSe carrier dynamics associated with the addition of hexadecylamine (HDA) as a cosolvent was also examined. Compared to tri-n-octlyphosphine oxide (TOPO) only synthesized nanocrystals, $\tau_{1}$ was shown to increase, indicating a reduction in the number of available surface traps with the addition of HDA. Based on surface stoichiometry, TOPO only $\mathrm{CdSe}$ nanocrystals are Cd-rich, indicating the presence of Se dangling bonds. These Se dangling bonds can then act as hole traps on the surface. Although an amine binding to selenium is not likely, due to the tilting of the HDA chain, it is possible that the methylene protons on HDA interact with the dangling selenium bonds on the surface. This is a plausible interaction, as it is doubtful that there is any coupling between the amine hydrogen atoms and the dangling selenium bonds on the surface. These results were corroborated through molecular modeling (Figure 2b).

(a)

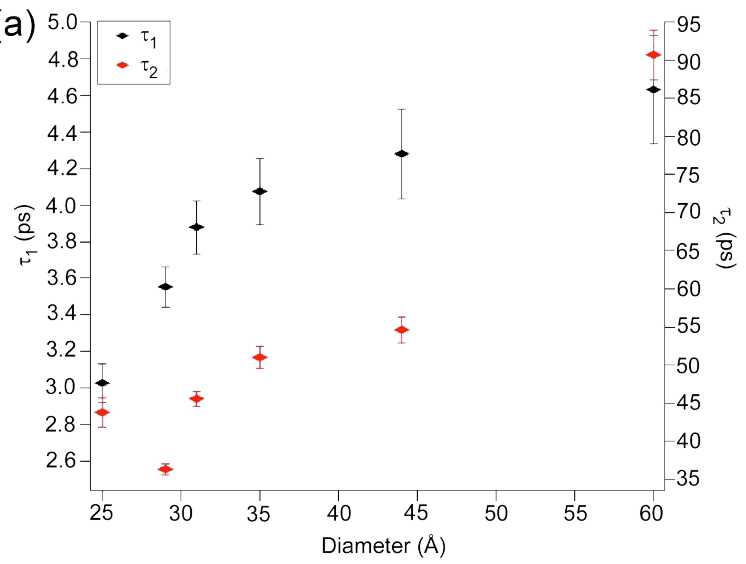

(b)

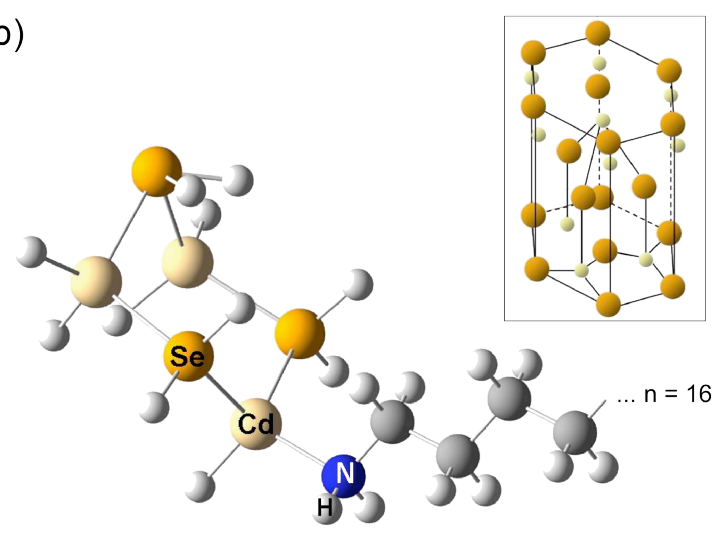

Figure 2. (a) Graph of $\tau_{1}$ and $\tau_{2}$ vs nanocrystal diameter. The increase in $\tau_{1}$ corresponds to the reduction in surface-to-volume ratio, resulting in a reduction in the overlap of the electron and hole wave functions with the nanocrystal surface. The increase in $\tau_{2}$ is attributed to the decrease in relaxation from a triplet state to the ground state and the increase in recombination through the transition from the singlet to the ground state as nanocrystal size increases. (b) GaussView3.09 model of a single surface slice of wurtzite $\mathrm{CdSe}$. The calculated distance between the amine hydrogen and the selenium atom was found to be $3.87 \AA$, whereas the characteristic bond length in free molecules for $\mathrm{H}-\mathrm{Se}$ is $1.47 \AA$. Consequentially, it is unlikely that there is any binding or coupling between the amine hydrogen and the selenium atoms. (Inset) CdSe wurtzite unit cell, where cations are represented as the small shaded circles. 
It has also been shown that photo-oxidation of the selenium surfaces of the nanocrystal leads to an increase in radiative decay efficiency from both the band edge and deep trap emission states (Figures $3 a$ and $3 b$ ). It has been further demonstrated that $\mathrm{CdSe} / \mathrm{ZnSe}$ core/shell nanocrystals are not immune from contributions from surface states because of the alignment of the band structures of the core and shell materials due to the band offsets (Figures $3 \mathrm{c}$ and $3 \mathrm{~d}$ ). ${ }^{3}$ This property of preferentially confining charges could possibly be exploited in tailoring a material for use in electronic devices such as photovoltaics.

(a)

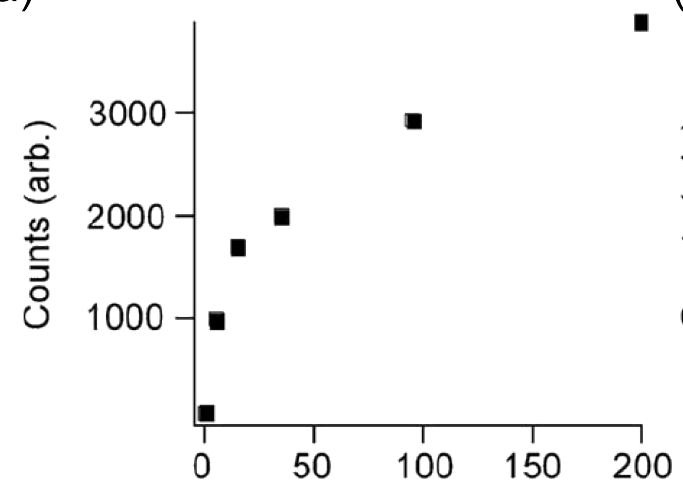

(c)

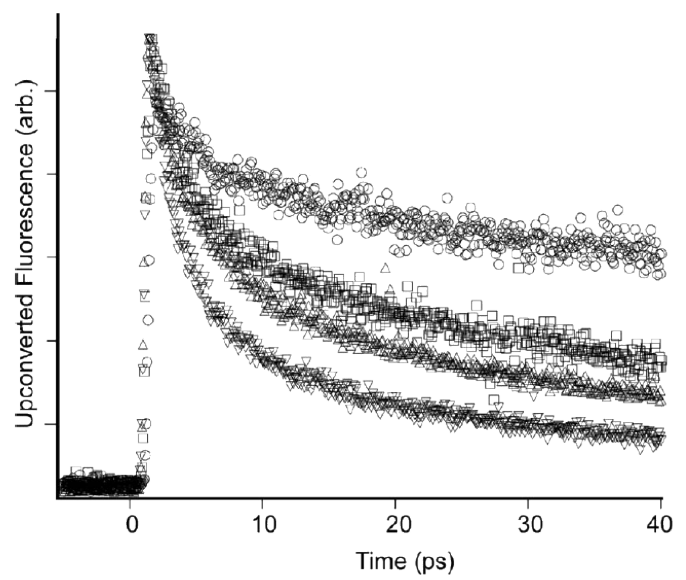

(b)

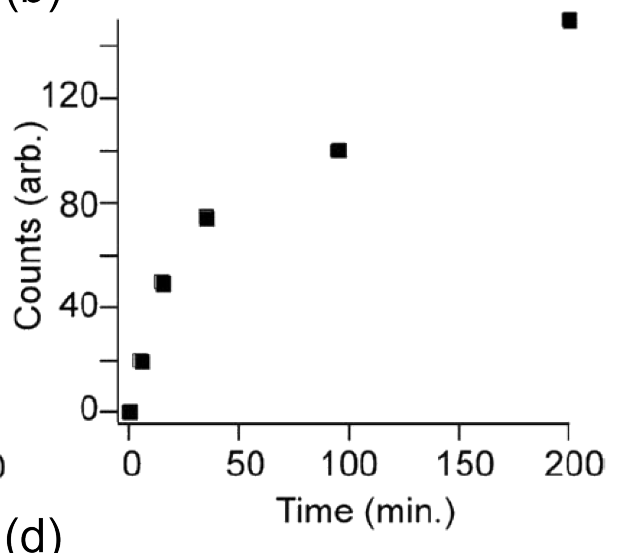

(d)

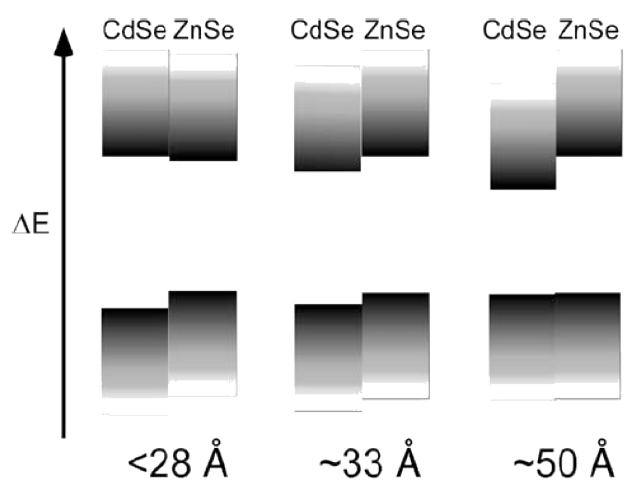

Figure 3. Bandedge emission (A) and deep trap emission (B) monitored as a function of oxygen exposure time. (C) Ultrafast fluorescence upconversion spectra of nanocrystals of all surface treatments studied with a $560 \mathrm{~nm}$ band edge absorption feature. Washed $\mathrm{CdSe} / \mathrm{ZnSe}$ core/shells (circles), unwashed $\mathrm{CdSe} / \mathrm{ZnSe}$ (squares), TOPO/HDA CdSe nanocrystals (triangles), and TOPO only CdSe nanocrystals (inverted triangles). (D) Band offsets between different sized CdSe core nanocrystals and the $\mathrm{ZnSe}$ shell.

\section{Band Edge Recombination in CdSe, CdS and CdSxSe1-x Alloy Nanocrystals Observed by Ultrafast Fluorescence Upconversion: The Effect of Surface Trap States}

The effect of surface trap states on band edge recombination in CdSe, $\mathrm{CdS}$ and $\mathrm{CdS}_{x} \mathrm{Se}_{-x}$ alloy nanocrystals has been determined using fluorescence upconversion spectroscopy. These measurements revealed both a size and composition dependence of the short-lived 
$(\tau 1)$ and long-lived $(\tau 2)$ components of fluorescence lifetime at the band edge. Increasing the nanocrystal diameter, $(2.3$ to $6.0 \mathrm{~nm})$, increases $\tau 1$. This is explained by the decrease in accessible trap sites through a reduction in surface-to-volume ratio. $\tau 2$ is found to also increase with increasing nanocrystal diameter. However, increasing the sulfur concentration in alloy nanocrystals, results in both a reduction in the magnitude of $\tau 1$ and a reversal in the trend for $\tau 2$. These changes in lifetimes associated with the addition of sulfur are explained by increased surface trapping. These results indicate that carrier dynamics may be controlled not only through size, but also through composition of the nanocrystals. Additionally, an increase in the Stokes shift is observed for $\mathrm{CdS}_{x} \mathrm{Se}_{1^{-x} x}$ alloy nanocrystals as compared to CdSe and CdS nanocrystals. This indicates that the Stokes shift is highly influenced by the nonlinear effects of alloying.
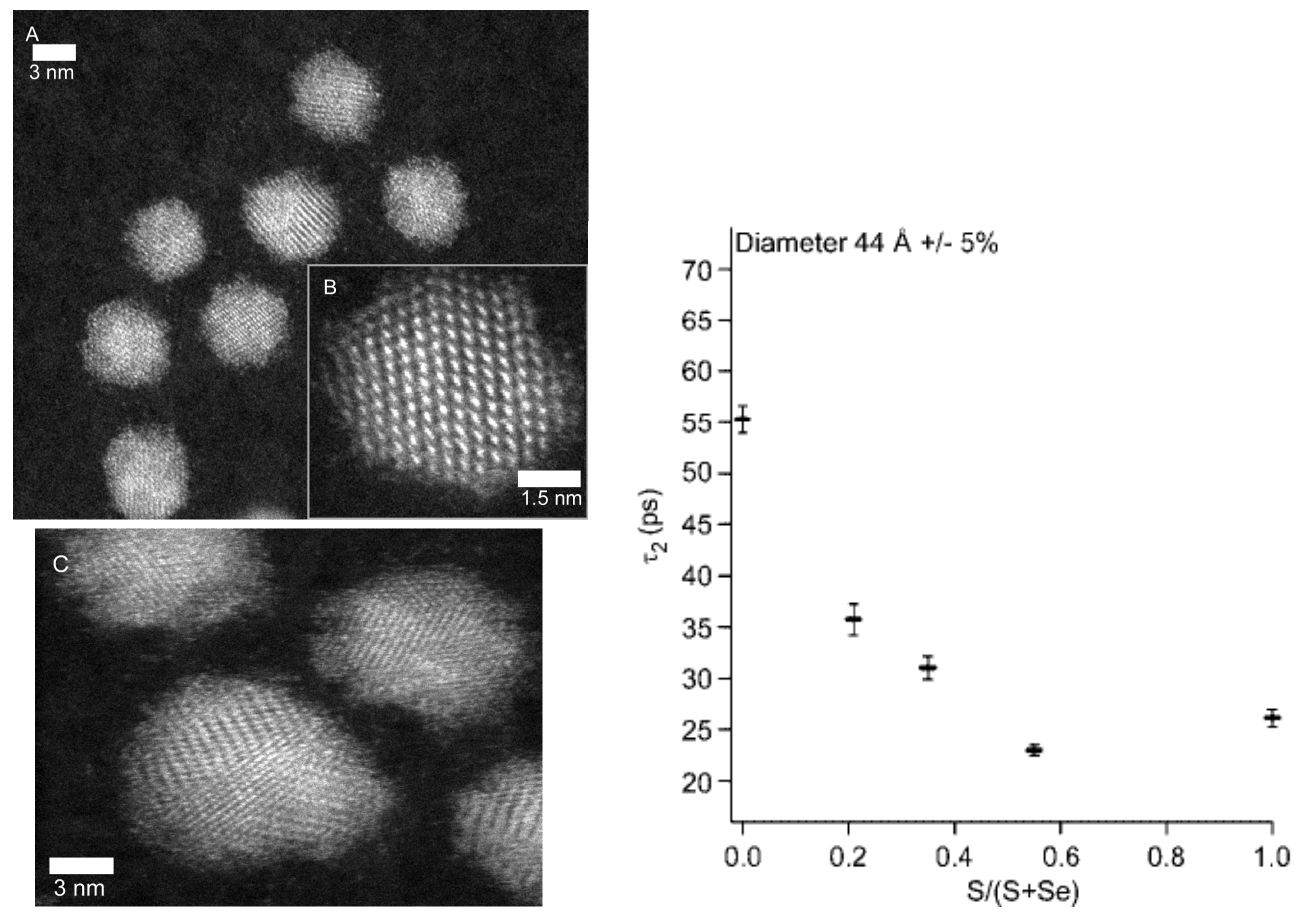

Figure 4. (a) Z-STEM image of CdS0.50Se0.50 $51 \AA$ alloy nanocrystals taken on a VG Microscopes' model HB603U, operating at $300 \mathrm{kV}$ and fitted with a Cs corrector from Nion, located at Oak Ridge National Laboratory. (b) It can be seen that there is no core/shell structure, as the nanocrystal is uniform in intensity. Right: Graph of $\tau 2$ versus nanocrystal composition for a series of $44 \AA$ ( $(5 \%)$ alloy nanocrystals. It can be seen that $\tau 2$ begins to decrease in the $\mathrm{CdS} x \operatorname{Se} 1-x$ alloy material. The decrease in $\tau 2$ with increasing sulfur contribution is attributed to increased trapping at the nanocrystal surface.

\section{Pinned Emission in Ultrasmall Nanocrystals.}

The emission spectrum for ultrasmall CdSe nanocrystals, passivated with alkyl phosphonic acids, with a diameter of $1.7 \mathrm{~nm}$ and smaller is shown to be pinned at 440 $\mathrm{nm} .{ }^{5}$ When the surface ligand is changed from a phosphonic acid to either dodecanethiol, oleic acid or pyridine, the emission at $440 \mathrm{~nm}$ is quenched. However, the positions of the remaining features are preserved, except in the case of the $550 \mathrm{~nm}$ emission for 
dodecanethiol. This indicates the pinning of the $440 \mathrm{~nm}$ emission peak is due to a surface state introduced by the phosphonic acid ligand binding to the surface of the nanocrystal.
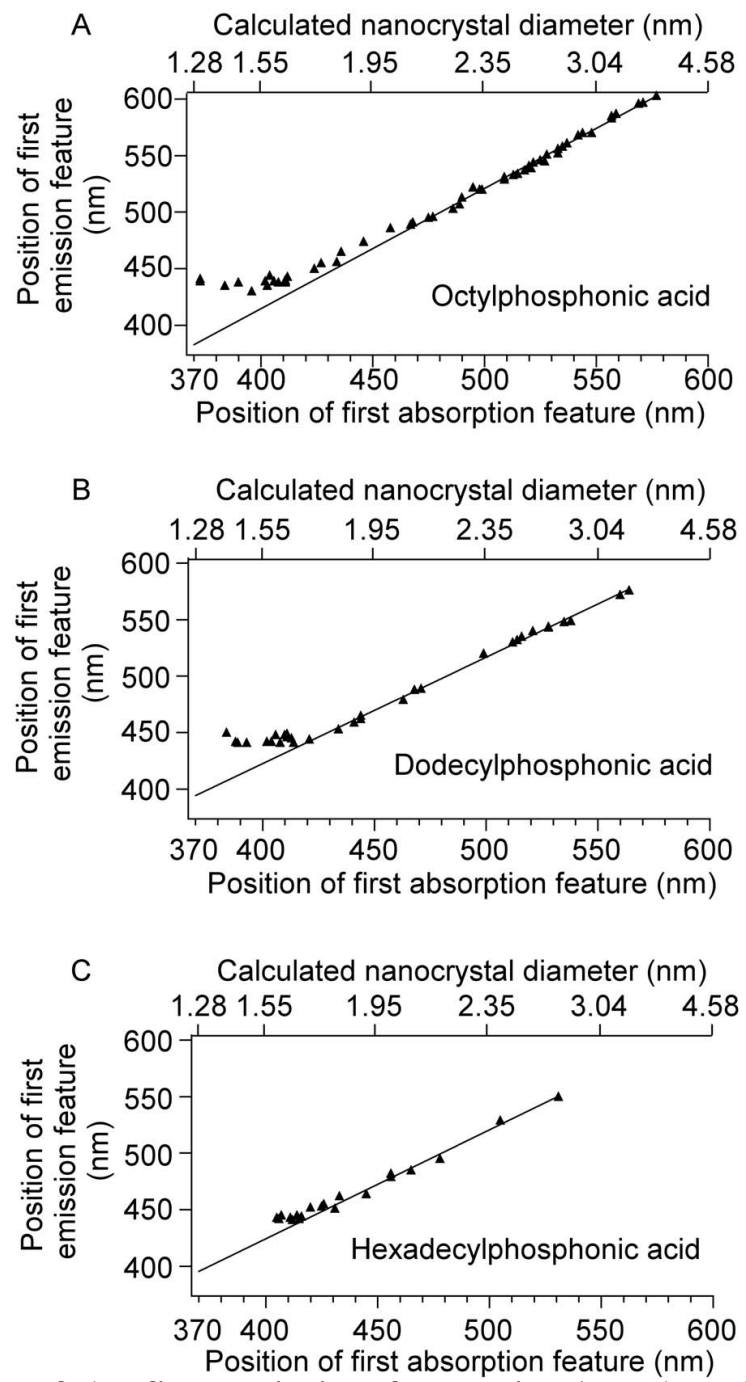

Figure 5. The position of the first emission feature is plotted against the position of the first absorption feature for CdSe nanocrystals passivated with (a) octylphosphonic acid, (b) dodecylphosphonic acid, and (c) hexadecylphosphonic acid. In all cases, when the band edge absorption wavelength is below $420 \mathrm{~nm}$, corresponding to nanocrystals less than $1.72 \mathrm{~nm}$ in diameter, the first emission feature is pinned at approximately $440 \mathrm{~nm}$. The line on each graph represents the trend of blue-shifting emission with a blue-shift in the absorption. The emission spectrum remains broad and unchanged at nanocrystal sizes below $2 \mathrm{~nm}$.

\section{Control of Surface State Emission via Phosphonic Acid Modulation in Ultrasmall CdSe Nanocrystals: The Role of Ligand Electronegativity}

In addition to showing that the emission for CdSe nanocrystals becomes pinned at ultrasmall size, we have also demonstrated the ability to modify this pinned emission from 2.79 to $2.91 \mathrm{eV}$ by changing the phosphonic acid ligand. ${ }^{6} \mathrm{~A}$ blue shifting emission 
is observed and is attributed to the increased electronegativity of shorter chain length ligands. Additionally, the quantum yield of these nanocrystals increases with increasing ligand chain length. This data further solidifies the critical role surface ligands play on the emission properties of ultrasmall nanocrystals.
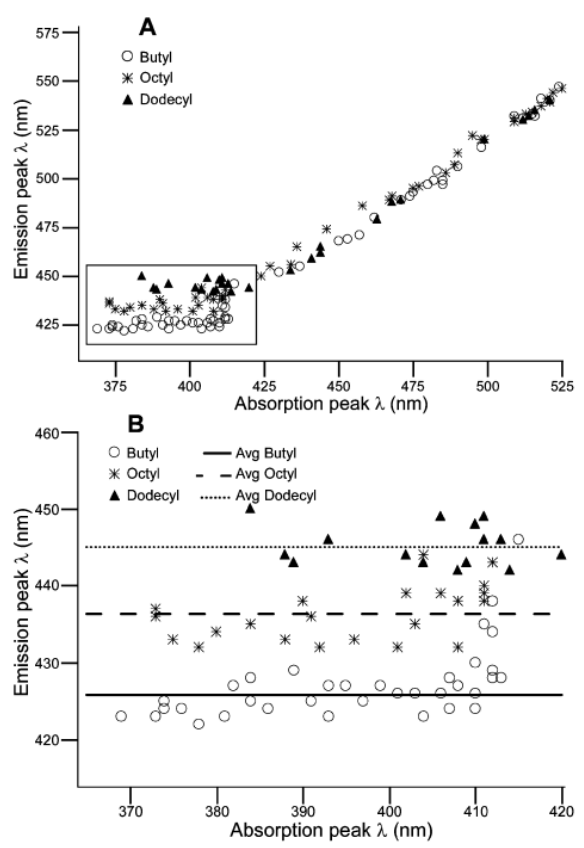

Figure 6. Modulation of white light CdSe surface-emission via ligand electronegativity. ${ }^{6}$

\section{Encapsulated White-light CdSe Nanocrystals as Nanophosphors for Solid-state Lighting}

In order to utilize ultrasmall nanocrystals in devices, a suitable encapsulant needed to be determined that did not cause them to aggregate and that does not alter the emission color. Ultrasmall CdSe nanocrystals were encapsulated in a variety of polymers. ${ }^{7} \mathrm{~A}$ correlation between the Hildebrand parameter and the Hildebrand solubility parameters of the encapsulants and their effectiveness was found, which was also dependent on the nanocrystal ligands BP-PFCB was found to offer a practical option for encapsulation, creating a rugged, color-stable environment for the white-light nanocrystals. This encapsulation in BP-PFCB allowed the nanocrystals to be combined with UV LEDs to create a potentially viable, solid-state white-light source. Initial attempts at coating UV LEDs exhibited CIE chromaticity coordinates $(0.324,0.322)$ and a CRI of 93. 

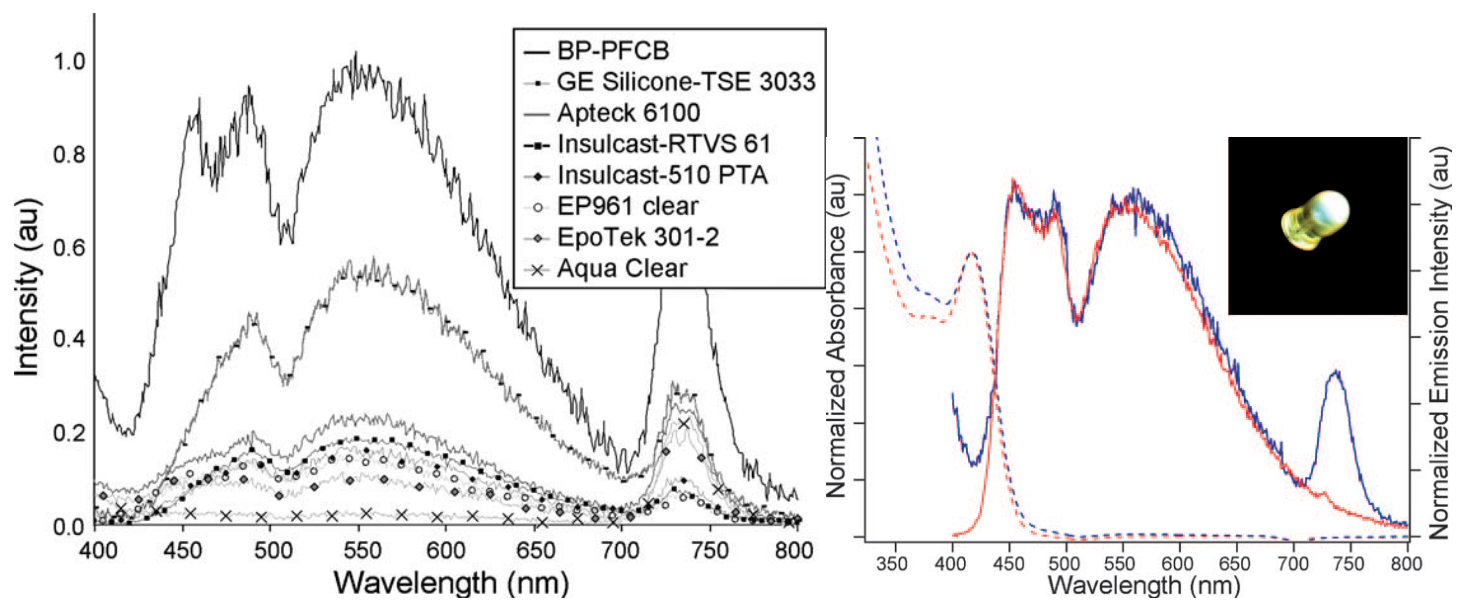

Figure 7. Identification of a suitable encapsulant for ultrasmall CdSe nanocrystals for white light applications. ${ }^{7}$

\section{White Light-emitting Diodes Based on Ultrasmall CdSe Nanocrystal Electroluminescence}

Prototypical electroluminescent devices were fabricated utilizing the native white-light emission for ultrasmall nanocrystals to produce a white light LED. ${ }^{8}$ This was the first electroluminescent device produce using nanocrystals with diameters less than $2 \mathrm{~nm}$. The LEDs had excellent color characteristics, with CIE color coordinates $(0.333,0.333)$, correlated color temperatures of 5461-6007 K, and color rendering indexes as high as 96.6 .
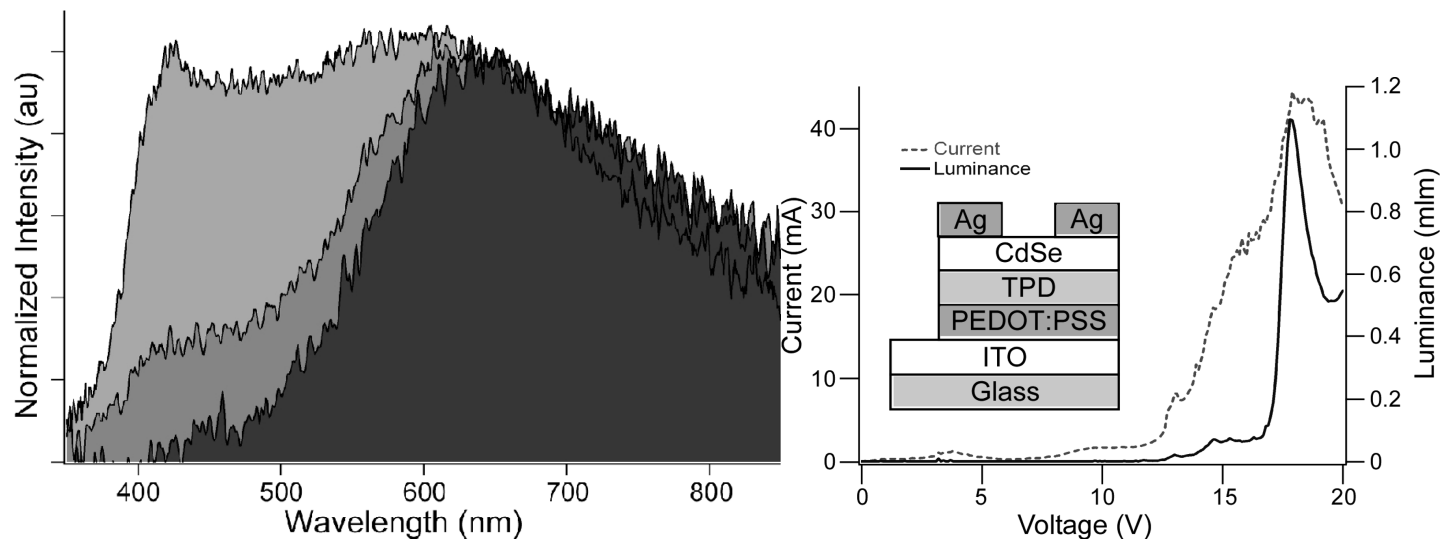

Figure 8. White light from electroluminescent device based off of ultrasmall CdSe nanocrystals. $^{8}$

\section{Structure and Ultrafast Dynamics of White Light-emitting CdSe Nanocrystals}

We obtained the first atomic resolution images as well as the first ultrafast fluorescence upconversion spectra of ultrasmall CdSe nanocrystals. ${ }^{9}$ The aberration-corrected STEM 
image indicated that they are crystalline, and consist of approximately four lattice planes with defects. The different ultrafast decay rates measured indicate that a variety of trapmediated pathways are responsible for the radiative recombination and involve both the electron and the hole.
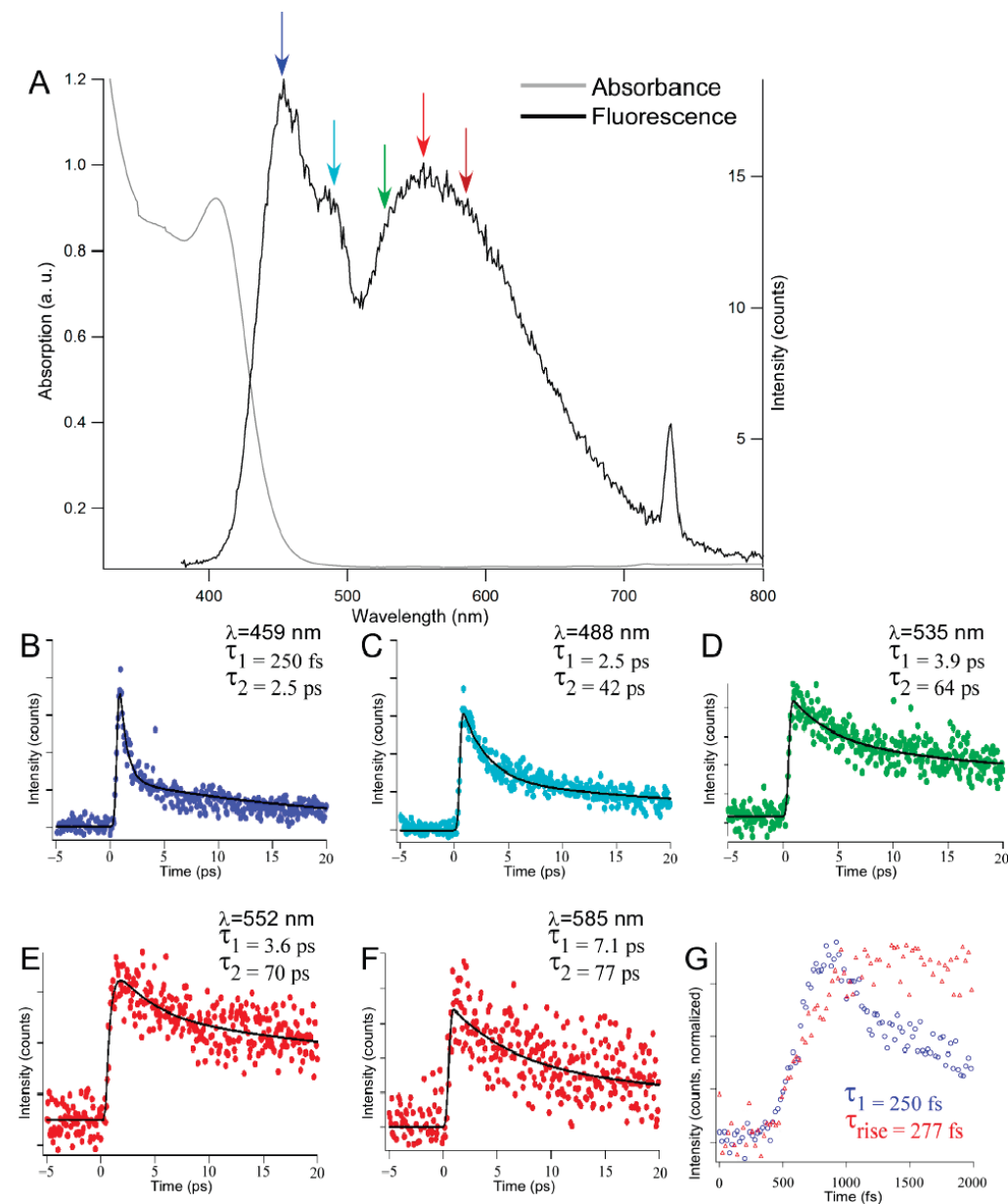

Figure 9. Ultrafast dynamics of ultrasmall CdSe nanocrystals. (A) Absorption and emission of a typical sample exhibiting a narrow band-edge absorption (light-gray line) and white-light emission (black line). The colored arrows indicate the wavelengths probed during the ultrafast experiment. (B-F) ultrafast data traces acquired at (B) 459, (C) 488, (D) 535, (E) 552, and (F) $585 \mathrm{~nm}$. (G) Comparison of the early time dynamics at 459 and $552 \mathrm{~nm}$. The fast decay at $459 \mathrm{~nm}$ (blue circles) coincides with the rise occurring at $552 \mathrm{~nm}$ (red triangles), indicating that the state emitting at $585 \mathrm{~nm}$ is being populated by the state emitting at $459 \mathrm{~nm}$.

\section{References:}

1. Smith, N.J.; Emmett, K.J.; Rosenthal, S.J. Photovoltaic cells fabricated by electrophoretic deposition of CdSe nanocrystals Appl. Phys. Lett. 2008, 93 (4).

2. Garrett, M.D.; Bowers II, M.J.; McBride, J.R.; Orndorff, R.L.; Pennycook, S.J.; Rosenthal, S.J. Band Edge Dynamics in CdSe Nanocrystals Observed by Ultrafast Fluorescence Upconversion J. Phys. Chem. C 2008, 112 (2), 436-442. 
3. Kippeny, T.C.; Bowers, M.J.; Dukes, A.D.; McBride, J.R.; Orndorff, R.L.; Garrett, M.D.; Rosenthal, S.J. Effects of surface passivation on the exciton dynamics of CdSe nanocrystals as observed by ultrafast fluorescence upconversion spectroscopy J. Chem. Phys. 2008, 128 (8).

4. Garrett, M.D.; Dukes, A.D.; McBride, J.R.; Smith, N.J.; Pennycook, S.J.; Rosenthal, S.J. Band edge recombination in $\mathrm{CdSe}, \mathrm{CdS}$ and $\mathrm{CdS}_{\mathrm{x}} \mathrm{Se}_{1-\mathrm{x}}$ alloy nanocrystals observed by ultrafast fluorescence upconversion: The effect of surface trap states J. Phys. Chem. C 2008, 112 (33), 12736-12746.

5. Dukes III, A.D.; Schreuder, M.A.; Sammons, J.A.; McBride, J.R.; Smith, N.J.; Rosenthal, S.J. Pinned Emission from Ultrasmall Cadmium Selenide Nanocrystals J. Chem. Phys. 2008, 129, 121102.

6. Schreuder, M.A.; McBride, J.R.; Dukes, A.D.; Sammons, J.A.; Rosenthal, S.J. Control of Surface State Emission via Phosphonic Acid Modulation in Ultrasmall CdSe Nanocrystals: The Role of Ligand Electronegativity J. Phys. Chem. C 2009, 113 (19), 8169-8176.

7. Schreuder, M.A.; Gosnell, J.D.; Smith, N.J.; Warnement, M.R.; Weiss, S.M.;

Rosenthal, S.J. Encapsulated white-light CdSe nanocrystals as nanophosphors for solid-state lighting J. Mater. Chem. 2008, 18 (9), 970-975.

8. Schreuder, M.A.; Xiao, K.; Ivanov, I.N.; Weiss, S.M.; Rosenthal, S.J. White Light-Emitting Diodes Based on Ultrasmall CdSe Nanocrystal Electroluminescence Nano Lett. 2010, 10 (2), 573-576.

9. Bowers II, M.J.; McBride, J.R.; Garrett, M.D.; Sammons, J.A.; Dukes III, A.D.; Schreuder, M.A.; Watt, T.L.; Lupini, A.R.; Pennycook, S.J.; Rosenthal, S.J. Structure and Ultrafast Dynamics of White-Light-Emitting CdSe Nanocrystals $J$. Am. Chem. Soc. 2009, 131 (16), 5730-5731. 